\title{
Symposium
}

\section{Sozialkonstruktivismus und Funktionalismus. Zwei problematische Figuren in Gesa Lindemanns Gesellschaftstheorie}

Symposiumsbeitrag zu: Gesa Lindemann, Strukturnotwendige Kritik. Theorie der modernen Gesellschaft. Band 1. Weilerswist: Velbrück Wissenschaft 2018, 456 S., kt., $25,00 €$

Besprochen von PD Dr. Jens Greve: Wissenschaftlicher Mitarbeiter, Institut für Soziologie, GeorgAugust-Universität Göttingen, E-Mail: jensgreve@gmx.de

https://doi.org/10.1515/srsr-2020-0021

Schlüsselwörter: Relationale Sozialtheorie, Gesellschaftskritik, Relationales Subjekt

Lindemanns sozialtheoretischer Ausgangspunkt ist die These der Kontingenz der Mitwelt. Die damit aufgeworfene Frage, wer als legitimer Akteur überhaupt gelten kann, beschäftigte sie bereits in vorangegangenen Büchern (Lindemann, 2009; 2014), auf deren Befunde sie zurückgreift. Die Annahme der Kontingenz der Mitwelt ist aber nicht nur ein Ausgangspunkt, sondern führt auf zwei zentrale Thesen, welche auch die Gesellschaftstheorie in ein anderes Licht rücken sollen. Lindemann stellt fest, dass die Frage, welche Akteure als legitime Akteure gelten können, in der Soziologie zu selten gestellt wurde und dass sich - dies ist die erste These - zeigen lässt, dass die Beschränkung auf lebendige Menschen, welche in der gängigen Sozial- und Gesellschaftstheorie vorgenommen wird, sich als eine historisch kontingente Betrachtungsweise darstellt. Ausgehend von dieser sozialtheoretischen Annahme ist die zweite, gesellschaftstheoretische These, dass die Beschränkung des Kreises legitimer Akteure auf lebendige Menschen und der damit verbundene Ausschluss von Tieren, Ahnen, Göttern und Geistern nicht zufällig einhergeht mit dem Übergang zur modernen Gesellschaft. „Gemäß der hier vorgeschlagenen Theorie [...] bildet die horizontal differenzierte Gesellschaft die erste Gesellschaft, die aus Menschen besteht“ 
(306). ${ }^{1} \mathrm{Zu}$ den Kennzeichen dieser Gesellschaft gehöre es, dass sie von einer gleichen Würde aller Personen ausgehe und entsprechend die Menschenrechte als normatives Fundament begreife.

Die Verbindung der beiden Thesen wirft eine zentrale Frage auf: Wenn die Grenzen der Sozialwelt ontologisch betrachtet kontingent sind, wieso muss diese Grenze sich in der Moderne in einer ganz besonderen Weise ausformen? Es könnte sich ja auch um eine historisch kontingente Setzung handeln, die mit den Strukturen der Gesellschaft nur zufällig zusammenhängt. Lindemann greift zwar zurecht auf die These Durkheims zurück, dass die moderne differenzierte Gesellschaft das Menschsein als einziges verbindendes Band zurücklasse, aber dies beantwortet noch nicht die Frage, warum es ausschließlich lebendige Menschen sein sollen, denen der Anspruch auf gleiche Rechte, Würde und individuelle Freiheit zugestanden wird - wieso sollten in der Moderne nicht auch andere Wesen als gleichberechtigte Wesen gelten können?

Diese Frage lässt sich meines Erachtens nicht in der Weise beantworten, wie Lindemann es versucht, und dass dies so ist, ergibt sich aus zwei problematischen Theoriefiguren, einem radikalen Konstruktivismus einerseits und einer funktionalistischen Notwendigkeitsbehauptung andererseits. Radikal konstruktivistisch ist die These von Lindemann, weil sie davon ausgeht, dass die Zulassung zum Kreis legitimer Akteure sich nicht auf eine ontologische Differenz zurückführen lässt, sondern auf Anerkennungsordnungen, die triadisch verstanden werden müssten. „Denn erst die Vermittlung durch Dritte erlaubt die Unterscheidung zwischen personalen und anderen Entitäten. Gewalt findet immer in einer drittenvermittelten Verfahrensordnung statt, durch die festgelegt wird, [...] wie der Kreis der legitimen Personen zu begrenzen ist“ (176, vgl. auch 16, 18, 23ff., 53, 56, 176ff.). Der ontologischen Frage lässt sich freilich durch eine Anerkennungsthese nicht ausweichen, denn die Anschlussfrage an die Anerkennungsthese lautet, wer derjenige ist, der anerkennt oder anerkennungsfähig ist. Sind die Autorinnen und Autoren der modernen Verfahrensordnung Menschen oder auch nicht-menschliche Wesen? Konnten die nicht-menschlichen Wesen zuvor Autorinnen und Autoren der entsprechenden Verfahrensordnungen sein? Die These, dass die Frage, wer legitimer Sprecher sein kann, durch eine Drittenkonstellation geklärt wird, führt im Übrigen in einen unendlichen Regress: Damit der Dritte festlegen kann, wer als legitimer Akteur gelten kann, muss seine Legitimität ja bereits durch einen weiteren Dritten bestätigt worden sein, für den sich freilich wiederum die Legitimationsfrage stellt, usw. (Greve, 2012; 2015a).

1 Seitenangaben ohne weitere Informationen beziehen sich auf den besprochenen Band. 


\section{Die Kontingenz der Mitwelt und die Moderne}

Lindemann ist darin zuzustimmen, dass die Frage nach den Grenzen der Sozialwelt in der Soziologie kaum thematisiert wurde - was sich bekanntlich erst mit Latour schlagartig geändert hat. Das aber heißt nicht, dass die Frage ignoriert wurde. Weber z.B. begründet die Festlegung auf Menschen dadurch, dass allein diese sich in sinnvermittelter Weise aufeinander beziehen - zumindest soweit die Forschung dies auch aus empirischen Gründen für zutreffend hält (Weber, 1980: 7). An anderer Stelle habe ich diese These auch vor dem Hintergrund aktueller Forschung verteidigt (Greve, 2011; 2015b). Bislang gibt es immer noch gute Gründe, das Wesen der humanspezifischen Sozialität darin zu sehen, dass allein lebendige Menschen sich in ihrem Verhalten nicht nur an den Erwartungen anderer orientieren, sondern davon ausgehen, dass sie wechselseitig darum wissen, dass die jeweils andere Person sich an den eigenen Erwartungen ebenfalls orientieren kann, m.a.W. dass sie über ein Bewusstsein der doppelten Kontingenz verfügen. ${ }^{2}$

Nun widerspricht diese Empirie auf den ersten Blick einer anderen Empirie, der ethnologischen, auf die Lindemann sich beruft. Neben Leenhardt sind es v.a. Descola und Viveiros de Castro, welche als Gewährsmänner auftreten. Die von ihnen beschriebenen Gemeinschaften sind bevölkert von beseelten Tieren und wirkmächtigen Ahnen - hier existiere (noch) die Idee einer great-chain-of-being (Lovejoy), die dann im Verlauf der Durchsetzung der Moderne durchschnitten werde (114ff.).

Lindemann zeigt zwar anhand einer Reihe von Beispielen, dass bis in die frühe Neuzeit hinein Verunsicherungen im christlichen Europa darüber herrschten, welchen Status der leibliche Körper, Hexen und Dämonen besaßen, dennoch wird man dem Christentum eine anthropozentrische Grundhaltung durchaus attestieren können. Das Christentum verdrängt eine Pluralität von Geistern und die Tiere werden in der Hierarchie des Seins nach unten verschoben. Wenn nur den Menschen unsterbliche Seelen zukommen (wie bei Augustinus und Thomas von Aquin), so bezeichnet dies eine operativ natürlich entscheidende Differenz. Auch die Bibel enthält eindeutig anthropozentrische Momente. Dies gilt nicht nur für den Herrschafts- oder Verantwortungsanspruch, sondern auch für die Idee der Ebenbildlichkeit, die dem Menschen zukommt (Genesis 1, 26ff.).

Sicherlich wird man konzedieren können, dass der Übergang zur modernen, funktional differenzierten Gesellschaft mit der Zurückdrängung der Religion ver-

2 „Andere Primaten strukturieren ihre Kommunikation nicht auf dieselbe Weise [wie Menschen] durch gemeinsame Absichten, gemeinsame Aufmerksamkeit, wechselseitig vorausgesetzte Kooperationsmotive und Kommunikationskonventionen. Vielmehr versuchen sie schlicht, die individuellen Ziele, Wahrnehmungen und Handlungen anderer vorherzusagen oder zu manipulieren“ (Tomasello, 2009: 120). 
bunden ist, was aber keineswegs heißt, dass der Glaube an Gott oder Götter verloren gegangen ist - so geben beispielsweise 2001 72\% der Befragten in 18 Ländern, die mehrheitlich der OECD angehören, an, dass sie an Gott glauben (Norris/Inglehardt, 2011:90). Säkularisierung meint zwar die Trennung von Staat und Kirche, nicht aber den Abschied vom Glauben an Gott oder Götter (Casanova, 1994). Wieso aber konnte Gott nicht als legitimes politisches Mitglied in die moderne Gesellschaft aufgenommen werden? Die naheliegende Antwort liegt darin, dass mit dem Glauben an Gott der Glaube an eine höhere Autorität verbunden war, die alle menschlichen Belange zu regeln in der Lage und befugt ist, und der daher einer Autonomisierung verschiedener Funktionsbereiche im Weg stand. Liest man es so, zeigt sich aber, dass nicht die Existenz mannigfaltiger Wesen dysfunktional im Hinblick auf die Durchsetzung funktionaler Differenzierung ist, sondern der Anspruch auf Suprematie, der für eines dieser Wesen behauptet wurde. Der damit einhergehende Suprematieanspruch der Kirche erwies sich nicht nur als hinderlich im Hinblick auf die Entwicklung nicht-religiösen Wissens, sondern auch als problematisch im Hinblick auf plurale Weltdeutungen (Berger, 1994). Die Frage nach der funktionalen Bedeutung einer Einschränkung der legitimen Wesen lässt sich daher durch diesen Prozess der Säkularisierung nicht erklären, dieser erklärt nur die Abkehr von Suprematieansprüchen - eine Wiederkehr vieler Götter erscheint ebenfalls denkbar.

Lindemanns grundlegende sozialtheoretische Annahme, dass es sich bei der Kontingenz der Mitwelt um eine historisch variable Zulassung und Ausschließung von Akteuren handele, steht vor einer weiteren Herausforderung. So weist Lindemann am Ende ihres Buches zu den Weltzugängen auf ein Problem der Erfassung der entsprechenden Prozesse hin: „Obwohl die Theorie davon ausgeht, dass es historisch kontingent ist, welche Wesen z.B. als zentrisch oder exzentrisch positioniert erscheinen, folgt die Anordnung der Beispiele der Strukturvorgabe des modernen historischen Apriori. Demzufolge werden differenzierte sprachlich vermittelte kulturelle Bildungen operativ von Menschen erzeugt, aber nicht von Geistern, Ameisen, Pavianen oder Göttern. [...] Gegen ihre eigene Intuition wiederholt die Theorie damit in ihrer operativen Ausarbeitung den blinden Fleck der Moderne. Immerhin: Die Reflexivität der Theorie erlaubt es, dies festzustellen“ (Lindemann, 2014: 332). Wenn dies aber der blinde Fleck der Moderne wäre, dann müssten sich vormoderne Zeugnisse eines nicht-menschlich erfahrenen Weltzugangs aufspüren lassen. Die Abwesenheit solcher Belege plausibilisiert eher die von mir präferierte Gegenthese, dass es nämlich letztlich nur durch und am Menschen ablesbare Handlungsstrukturen sind, welche die Vergesellschaftung tragen (Greve, 2015b). ${ }^{3}$

3 Interessanterweise kommt auch Descola zu einem ähnlichen Resultat: „In Amazonien, im subarktischen Amerika, in Nordsibirien werden die Bindungen, die die Tiere oder die Geister unter- 


\section{Gesellschaft}

Lindemanns Analyse der modernen Gesellschaft folgt dem von Durkheim, Weber, Simmel und Luhmann vorgezeichneten Weg der Annahme einer Differenzierung von sachlich disparaten Teilbereichen der Gesellschaft, die - und hier greift Lindemann auf Luhmann zurück - in einem nicht-hierarchischen Verhältnis zueinander stehen (138). Wie für Luhmann und für Habermas ist für Lindemann der Kommunikationsbegriff zentral, wenn er auch nicht als Grundbegriff ausgewiesen ist. Kommunikative Prozesse schlagen sich nicht zuletzt in legitimatorischen Diskursen nieder. Hier näher an Habermas, rückt die Legitimationsfrage bei Lindemann viel deutlicher ins Zentrum der Gesellschaftstheorie als bei Luhmann. Legitimationen verweisen auf Dritte (143f.) und die Drittenkonstellation wird für die Analyse des Differenzierungsmusters der Moderne verwendet (157ff.). ${ }^{4}$ Einerseits ließen sich gesellschaftliche Teilbereiche danach unterscheiden, ob sie mitgliedschaftlich verfasst sind (Politik, Recht) oder nicht (Wirtschaft, Wissenschaft). Quer dazu liegt die Unterscheidung zwischen anwesenden (oder zukünftig anwesenden) und abwesenden Dritten. Diese Unterscheidung identifiziert Lindemann mit der von Lernen und normativem Erwarten. Die abwesenden Dritten dienen in der Politik als Garanten eines normativen Erkenntnisstils und dieser schlägt sich auch in Legitimationsformen für die weltgesellschaftlich (nicht mitgliedschaftlich) verfassten Bereiche nieder - und zwar in den Formen institutionalisierter Weltnormen, ,die sich durch den Bezug auf die Menschheit als dauernde Institution legitimieren“ (173) und die durch „Staaten, internationale Organisationen“ sowie „informelle Stellvertreter (NGOs, soziale Bewegungen)“ getragen werden (173).

Den Gedanken, dass es abwesende, nicht anwesende Dritte sind, welche Legitimationen tragen, übernimmt Lindemann vom frühen Luhmann. Ganz eindeutig kann diese Zuordnung freilich nicht gelingen, weil es - wie Lindemann ja auch sieht - in der Politik eben immer auch darauf ankommt, dass die Ordnung durch die Anwesenden selbst bestätigt wird (z.B. in Wahlen, 174). Das Problem der Repräsentation kehrt hiermit zurück. Da der abwesende Dritte nicht sprechen kann, bedarf es der Interpreten. Nicht zufällig bezieht sich Lindemann hier insbesondere auf soziale Bewegungen, welche das Menschenrechtsethos tragen (31ff., 365ff.). Trägt man der Verkörperung in den anwesenden Akteuren Rechnung, verkompli-

einander vereinen, sowie die der Menschen stets durch ein Vokabular bezeichnet, das dem Register des Umgangs zwischen Menschen entstammt“ (Descola, 2013: 370).

4 Daneben findet sich eine handlungstheoretische Formulierung des Differenzierungsmusters, welche dieses aus verschiedenen Beschreibungen des „Worum-willens“ der Funktionsbereiche ableitet (146ff.). 
ziert sich freilich das Bild. Keineswegs verfolgen alle sozialen Bewegungen oder alle weltgesellschaftlich relevanten Akteure ein an Gleichheit der Würde orientiertes Ethos der Menschrechte. Die Annahme, dass dieses Ethos gleichsam selbstverständlich das normative Fundament der Moderne darstellt, droht am Ende in den Platonismus der Weltkulturthese von Meyer et al. zu führen (Holzinger, 2018). Da dieses Ethos keineswegs als fraglos durchgesetztes gelten kann, stellt sich dringend die normative Frage, die, folgt man der Titelgebung dieses ersten Bandes, offensichtlich zentral ist.

\section{Kritik}

Das Buch nimmt die wieder virulenter gewordene Frage nach den Möglichkeiten einer kritischen Soziologie auf. Bekanntlich muss sich jede Kritik der Gesellschaft die Frage stellen, wie sie den Maßstab einer Kritik der Gesellschaft rechtfertigen kann. Lindemann folgt hier dem von Durkheim vorgezeichneten Weg: Die moralischen Überzeugungen und die Struktur der Gesellschaft stehen in einem nichtzufälligen Verhältnis. „Zwischen der neuen Form der Grenzziehung und der Entwicklung der Form horizontaler gesellschaftlicher Differenzierung gibt es einen wechselseitigen Bedingungszusammenhang“ (317, vgl. deutlich auch 319ff., 324, 334). Wer demnach die Struktur bewahren möchte, müsste auch die moralischen Werte aufrechterhalten und umgekehrt - entsprechend gehe es um die Identifikation von Selbstgefährdungen und ,gegebenenfalls [um, J.G.] eine praktische wirksame Gesellschaftskritik, um die Gesamtordnung horizontaler Differenzierung vor einer Selbstzerstörung zu bewahren“ (337). Zwei Probleme ergeben sich. Erstens setzt die These voraus, dass die Adressaten eines von beidem (Struktur oder Werte) möchten, sonst ergibt sich keine Notwendigkeit der Anpassung der Normen oder der Struktur. Zweitens ist fraglich, ob die zentrale Annahme, dass die moderne Gesellschaft dann und nur dann aufrechterhalten werden kann, wenn sie den Menschen die gleiche Würde zuspricht, überhaupt zutrifft. Dieser Nachweis könnte auf zwei Wegen geführt werden: zum einen auf einem sozio,logischen“ Weg, d.h. der Suche nach plausiblen sinnhaften Zusammenhängen. Durkheim und Luhmann bieten hier Argumente. Bei Ersterem lautet, wie bereits erwähnt, die Annahme, dass die Gleichheit der Individuen als letztes verbliebenes Band in der differenzierten Gesellschaft gelten kann, bei Letzterem, dass die Funktionssysteme von sich aus keinen Grund zur Exklusion kennen oder auch, dass diese aufgrund ihrer Expansionstendenzen auf Inklusion aller zielen (ähnlich ja bereits Parsons, 1986). Auf das Problem dieser Argumentationslinie bei Lindemann hatte ich bereits hingewiesen: Warum sollte dies nur mit leibhaftigen Menschen möglich sein? Neben dem sozio,,logischen“ Weg lässt sich auch empirisch argumen- 
tieren. Eine solche Argumentation setzt sich zwar dem Verdacht eines naturalistischen Fehlschlusses aus, wichtiger ist hier aber, dass sich empirische Gegenevidenzen finden lassen. Menschliches Leben besitzt faktisch keineswegs den gleichen Würdeschutz und -anspruch: Embryonen können „verworfen“ und Kinder können abgetrieben werden (Hoerster, 1991). Von einem umfassenden Schutz menschlichen Lebens kann in der modernen Gesellschaft keine Rede sein, und dies gilt auch dort, wo dem keine unmittelbaren Knappheitsbedingungen entgegenstehen. ${ }^{5}$ Bekanntlich ist in der bioethischen und politischen Debatte umstritten, ob der uneingeschränkte Lebensschutz allem menschlichen Leben oder nur selbstbewussten menschlichen Individuen zukommt. Während Lindemann auf dem Wege des philosophischen Proseminars Luhmann vorrechnet, er könne durch die implizite Festlegung auf sinnbegabte Menschen nicht ausschließen, dass „neurologisch schwersterkrankte lebendige Körper gekeult [...] werden“ müssten, „unabhängig davon, ob es sich um Rinder- oder Menschenkörper handelt“ (314), besteht sie auf der Möglichkeit eines funktionalistischen Nachweises für die Ausdehnung auf alle lebenden Menschen unabhängig davon, ob diese über Selbstbewusstsein verfügen oder nicht (326). Begründet wird dies von Lindemann durch ein „Gedankenexperiment“: „Wenn das aktuelle Kriterium der Zuerkennung des Status einer sozialen Person, nämlich lebendig zu sein, durch das Kriterium ersetzt würde, aktuell die Fähigkeit zu zeigen, rational zu sich Stellung zu nehmen und intentional handeln zu können, müssten all diejenigen, die diese Merkmale nicht aufwiesen, aus dem Kreis der Personen ausgeschlossen werden. Dies müsste politisch-rechtlich in der gleichen Weise strikt durchgesetzt werden, wie gegenwärtig die Gültigkeit des Kriteriums lebendiger Körper“ (326). Während die erste Folgerung allein definitorisch zwingend ist, ist die Prämisse der zweiten Folgerung, wie eben gezeigt, falsch: Nicht alles menschliche Leben besitzt den gleichen uneingeschränkten Würdeschutz. Dass die moderne Gesellschaft mit abgestuften Würdeverständnissen sehr wohl kompatibel ist, zeigt sich darüber hinaus auch daran, dass zwischen der Würde von Tieren und Steinen ja ebenfalls ein Unterschied gemacht wird. M.a.W, es bleibt auch hier wieder unklar, warum alle lebendigen Menschen und nur sie als die legitimen Träger gleicher Würde zu gelten haben sollen.

Unter dem Stichwort der Kritik möchte ich zwei weitere Beobachtungen anschließen. Erstens: Über weite Teile beschreibt Lindemann Kritik als Soziologie

5 Und: Aus der Sicht der Wissenschaft sind „überzählige“ Embryonen, an denen geforscht werden kann, offensichtlich „funktionaler“ als ein umfassendes Verbot der „verbrauchenden“ Embryonenforschung. 
der Kritik. Hier kann sie sich auf die Beobachtungen verlassen, die viele bereits gemacht haben, nämlich dass zahlreiche moderne Gesellschaften (wenn auch sicherlich nicht alle) institutionelle Garantien für die kritische Selbstbeobachtung der Gesellschaft besitzen (Öffentlichkeit, Zivilgesellschaft, soziale Bewegungen) (334ff.). All dies trifft zu, sagt aber wenig über „notwendige“ Kritik. Zweitens: Lindemanns zentrale Kritik an der kritischen Theorie besagt, dass diese sich nicht hinreichend über ihr eigenes Verhältnis zur Gewalt klargeworden sei (358). Indem sie die Dimension der Gewalt ausblende, bleibe der kritischen Theorie allein noch eine moralisierende Rede (364). Diesen Befund teile ich, führe ihn aber nicht auf ein behebbares Reflexionsdefizit der kritischen Theorie zurück, sondern auf den Umstand, dass es der kritischen Theorie nicht gelungen ist, einen Standpunkt zu begründen, der es erlauben würde, jenseits des ohnehin schon bestehenden Konsenses Gewalt als legitim überhaupt ausweisen zu können (Greve, 2015c). Wenn Lindemann Habermas vorwirft, er ziele auf die Überwindung jeder Gewalt (339), übersieht sie, dass Habermas diese frühe Institutionenkritik gegen eine Position eingetauscht hat, die ihren Frieden mit der staatlichen Gewalt gefunden hat (vgl. bspw. Habermas, 1992: 168). Solange die staatliche Gewaltordnung als legitime gelten kann, sehen weder Habermas noch Lindemann darin ein Problem. Auch die Reflexionsschleife über Gewalt führt Lindemann letztlich dahin, dass die Theorie lediglich das Selbstverständnis moderner Demokratien wiederholt, ohne dass eine eigenständige normative Begründung der Überlegenheit dieser Sozialformation geleistet wird.

\section{Schluss}

Lindemanns Buch enthält eine Vielzahl interessanter Beobachtungen und feingliedriger Debatten, die hier nicht gewürdigt werden können. Hinsichtlich der Zentralthesen des Buches lassen sich aber zwei Einwände formulieren. Erstens ist $\mathrm{zu}$ fragen, ob es nicht eine sozialtheoretisch bedeutsame ontologische Differenz zwischen Wesen gibt, welche die These der Kontingenz der Mitwelt einschränken muss. Bei aller Variabilität in der Anerkennung anderer Wesen gibt es doch einen Kern dessen, was Personalität ausmacht, der an menschlichen Eigenschaften abgelesen wird. Bei den von Descola beforschten Achuar aus dem Amazonasgebiet beispielsweise werden zwar auch Tiere als Personen wahrgenommen, aber nur Menschen sind „vollständige“ Personen (Descola, 2013: 22f.). Entscheidend für diese Vollständigkeit ist die Fähigkeit, gesehen werden $\mathrm{zu}$ können und sich sprachlich verständigen zu können (für Weiteres vgl. Greve, 2015b). Auch werden die Grenzziehungen von Menschen bezeugt - eine Schwierigkeit, welche auch Lindemann sah. Hier mag man einwenden, dass es unscharfe Ränder gibt: Spricht 
aus der Bibel nicht Gott? Die Unschärfe der Ränder lässt sich allerdings ebenfalls für die Moderne konstatieren. Auch in der Moderne glauben Menschen an die Existenz von Gott, Göttern und Seelen. Lässt sich also tatsächlich ein so deutlicher Bruch zwischen den Kontingenzen der Mitwelt vor der Moderne und in der Moderne konstatieren? Plausibel ist die Annahme eines Bruchs politischer Legitimitätsvorstellungen in der europäischen Moderne, welche die Politik aus der Bindung an religiöse Vorstellungen herauslöst.

Meine Kritik lautete zweitens, dass moderne demokratische Ordnungen zwar keine Suprematieansprüche bestimmter Wesen anerkennen, dass daraus aber nicht folgt, warum es für moderne Ordnungen funktional notwendig ist, nur lebendige Menschen als legitime politische Akteure zuzulassen. Die funktionalistische Abhängigkeitsthese (moderne Gesellschaft funktional abhängig von Begrenzung auf lebendige Menschen) lässt sich nicht belegen. Darüber hinaus kennen moderne Gesellschaften durchaus eine abgestufte Würde lebendiger Menschen. Lindemanns Funktionalisierung der Kontingenz der Mitwelt hat schließlich eine interessante Konsequenz. Während wir mit Latour gesprochen niemals modern gewesen sind (Latour, 2008), vermag Lindemanns moderne Gesellschaft, wenn die funktionale Abhängigkeitsthese stimmt, die Kontingenz der Mitwelt eben gerade nicht zur Geltung zu bringen. Könnte aber eine Gesellschaft, die Rindern und Affen dieselbe Würde zusprechen würde wie Neugeborenen (Singer, 1994), nicht (mehr) modern sein? Ich denke doch, denn diese Folgerung würde sich ja nur dann ergeben, wenn Lindemanns funktionalistische These wahr wäre.

\section{Literatur}

Berger, P. L. Sehnsucht nach Sinn. Glauben in einer Zeit der Leichtgläubigkeit; Campus: Frankfurt a. M./New York, 1994.

Casanova, J. Public Religions in the Modern World; Chicago University Press: Chicago, 1994.

Descola, P. Jenseits von Natur und Kultur; Suhrkamp: Berlin, 2013.

Greve, J. Menschliche Aktorenschaft. In Akteur - Individuum - Subjekt: Fragen zu,Personalität und ,Sozialität‘; Lüdtke, N.; Matsuzaki, H., Hrsg.; VS: Wiesbaden, 2011; pp 221-237.

Greve, J. Praxis - Zuschreibung - Objektivität. Kölner Zeitschrift für Soziologie und Sozialpsychologie 2012, 64, 431-454.

Greve, J. Reduktiver Individualismus - Zum Programm und zur Rechtfertigung einer sozialtheoretischen Grundposition; Springer VS: Wiesbaden, 2015a.

Greve, J. Sinnkriterien und Handeln. Zur sozialtheoretischen Zentralität menschlicher Handlungsfähigkeit. In Routinen der Krise - Krise der Routinen. 37. Kongress der Deutschen Gesellschaft für Soziologie in Trier 2014; Lessenich, S., Hrsg.; 2015b.http://publikationen.soziologie.de/index.php/kongressband_2014/article/view/136 (Zugriff Jan 7, 2020).

Greve, J. Gesellschaftskritik und die Krise der kritischen Theorie. In Routinen der Krise - Krise der Routinen. 37. Kongress der Deutschen Gesellschaft für Soziologie in Trier 2014; Lessenich, 
S., Hrsg.; 2015c.http://publikationen.soziologie.de/index.php/kongressband_2014/article/view/139 (Zugriff Jan 7, 2020).

Habermas, J. Faktizität und Geltung. Beiträge zur Diskurstheorie des Rechts und des demokratischen Rechtsstaates; Suhrkamp: Frankfurt a. M., 1992.

Hoerster, N. Abtreibung im säkularen Staat: Argumente gegen den § 218; Suhrkamp: Frankfurt a. M., 1991.

Holzinger, M. Warum die Weltgesellschaft nicht existiert. Kritische Reflexionen zu einigen empirischen und epistemologischen Problemen der Theorie der Weltgesellschaft. Kölner Zeitschrift für Soziologie und Sozialpsychologie 2018, 70, 183-211.

Latour, B. Wir sind nie modern gewesen: Versuch einer symmetrischen Anthropologie; Suhrkamp: Frankfurt a.M., 2008.

Lindemann, G. Das Soziale von seinen Grenzen her denken; Velbrück Wissenschaft: Weilerswist, 2009.

Lindemann, G. Weltzugänge. Die mehrdimensionale Ordnung des Sozialen; Velbrück Wissenschaft: Weilerswist, 2014.

Norris, P.; Inglehardt, R. Sacred and Secular. Religion and Politics Worldwide; Cambridge University Press: Cambridge et al., 2011.

Parsons, T. Gesellschaften: Evolutionäre und komparative Perspektiven; Suhrkamp: Frankfurt a. M., 1986.

Singer, P. Praktische Ethik; Reclam: Stuttgart, 1994.

Tomasello, M. Die Ursprünge der menschlichen Kommunikation; Suhrkamp: Frankfurt a.M., 2009.

Weber, M. Wirtschaft und Gesellschaft. Grundriß der verstehenden Soziologie; Mohr (Siebeck): Tübingen, 1980. 